\title{
206. Remark on Fixed Point of k-regular Mappings
}

\author{
By Haruo MAKI \\ Department of Mathematics, Wakayama University, Wakayama \\ (Comm. by Kinjirô KUnUGI, M. J. A., Sept. 13, 1971)
}

The main purpose of this paper is to answer the question raised in [4]. The dilation $D_{k}$ of Euclidean $n$-space $R^{n}$ defined by $x \mapsto k x$ for some $k \in(0,1)$ can be extended uniquely to the $n$-sphere, $S^{n}=R^{n} \cup\{\infty\}$. If $h$ is a homeomorphism of $S^{n}$ of the same topological type as $D_{k}$, then $h$ is regular except at two points. Kérekjartó [6], Homma and Kinoshita [2] showed the converse for $n=2, n=3$ respectively. Husch [3] extended Homma and Kinoshita's result for $n \geqq 6$. He [4] considered the topological characterization of the dilation in a separable infinite dimensional Fréchet space $E$ (i.e. in a separable infinite dimensional locally convex complete linear metric space).

In [4], Husch has the following theorems. Let $h$ be a homeomorphism of $E$ (with metric $d$ ) onto itself.

Theorem (Husch [4]). Suppose that $h$ is $k$-regular at each point of $E, 0<k<1$ (i.e. for each $\varepsilon>0$, there exists $\delta>0$ such that if $d(x, y)$ $<\delta$, then $d\left(h^{n}(x), h^{n}(y)\right)<k^{n} \varepsilon$ for each integer $\left.n\right)$.

(1) ([4], Proposition 6, p. 4) $h$ has at most one fixed point.

(2) ([4], Theorem 1, p. 2) If the fixed point set of h, Fix (h), is not empty, then $h$ has the topological type of a dilation $D_{k}$.

(3) ([4], Theorem 2, p. 2) If Fix (h) is empty, then $h$ has the topological type of a translation.

In this paper we prove the following:

Theorem 1. If $h$ is $k$-regular at each point of $E, 0<k<1$, then $h$ has a unique fixed point.

Hence we can eliminate the hypothesis that $F i x(h)$ be a non empty set in Husch's result (2).

Every separable infinite dimensional Fréchet space $E$ is homeomorphic to the countable infinite product of lines [1]. Hence $E$ is connected metric space. Thus we only show the following:

Lemma 2. Let $h$ be a k-regular mapping, $(0<k<1)$, of a complete, connected metric space $X$ onto itself. Then $h$ has a unique fixed point.

Before starting the proof, we recall the following definitions and some properties [5]. Let $h$ be a continuous mapping in a metric space $X$. If for each $\varepsilon>0$, there exists $n \in I^{+}$(positive integers) such that

$$
d\left(h^{m}(x), h^{m}(y)\right)<\varepsilon \quad \text { for all } m \geqq n,
$$


then $x$ and $y$ are said to be asymptotic under $h$. (Abbreviate $x \sim y$ ). Then $\sim$ is an equivalence relation on $X$. Let $X_{h}$ be the set of all equivalence classes. $\quad \bar{x}$ denotes the equivalence class of $x \in X$. The induced mapping $\bar{h}: X_{h} \rightarrow X_{h}$ is well defined as follows. For each $\bar{x} \in X_{h}, \bar{h}(\bar{x})$ $=\overline{h(x)}$. Then we have the following theorems.

Theorem 3 (Kashiwagi and Maki [5], Theorem 12, p. 7). Let X be a complete metric space. Then the continuous mapping h has a unique fixed point if and only if the induced mapping $\bar{h}$ has a unique fixed point.

Theorem 4 ([5], Theorem 13, p. 7). Let all assumptions of Theorem 3 hold. If $X_{h}$ is a singleton, then $h$ has a unique fixed point.

Proof of Lemma 2. Since $h$ is $k$-regular at each point $x$, there exists a $\delta(x)$-neighbourhood $B_{x}(\delta(x))$, with center $x$ and radius $\delta(x)$ such that

$$
\text { if } \forall y \in B_{x}(\delta(x)), \quad \text { then } x \sim y \text {. }
$$

Let $x$ be any point of $X$. By the above discussion, $\bar{x}$ is open in $X$. And $\bar{x}$ is not empty. Note that $\bar{x}$ is a closed set in $X$. For suppose $\left\{x_{n}\right\}$ is a sequence in $\bar{x}$ such that

$$
x_{n} \rightarrow a \text { as } n \rightarrow+\infty \text {. }
$$

Since $h$ is $k$-regular at $a$, then there exists an integer $N$ such that

$$
x_{n} \sim a \text { for all } n>N . \quad x, x_{n} \in \bar{x} \text { implies } x_{n} \sim x \text { for } n .
$$

Hence we have $x \sim a, a \in \bar{x}$. This implies $\bar{x}$ is closed in $X$. Since $X$ is connected, $X=\bar{x}$. Hence $X_{h}$ is a singleton. With the use of Theorem 4 , the proof is complete.

Q.E.D.

Remark 1. Theorem 1 is the answer to the question raised in [4]. The hypothesis that Fix $(h)$ be empty can never be satisfied in Husch's result (3). Hence that lines should be delated from the theorem (Theorem 2 [4]).

Now, suppose that there exists an everywhere dense subset $Y$ of $X$. We have the following:

Theorem 5. Let $f$ be a k-regular mapping of a complete metric space $X$ onto itself. If $Y_{f}$ is a singleton, then $f$ has a unique fixed point.

Proof. We show that $X_{f}$ is a singleton. Let $x$ be any point of $Y$, $y$ any point of $X-Y$. Then there exists a sequence $\left\{x_{n}\right\}$ of $Y$ such that

$$
\lim _{n \rightarrow \infty} x_{n}=y \text {. }
$$

Since $f$ is $k$-regular, $x_{n} \sim y$ for some integer $n$. Clearly $x_{n} \sim x$. Hence $x \sim y$ if $x \in Y, y \in X-Y$. Now let $x, y$ be any points of $X-Y$. Then there exist the sequences $\left\{x_{n}\right\},\left\{y_{n}\right\}$ such that

$$
\lim x_{n}=x \text { and } \lim y_{n}=y \text {. }
$$

Since $x_{n}, y_{n} \in Y, y, x \in X-Y$, then $x_{n} \sim x$ and $y_{m} \sim y$ for some integers $n, m$. Since $Y_{f}$ is a singleton, we have $x \sim y$. This implies $X_{f}$ is a 
singleton.

Q.E.D.

Remark 2. If one replaces the condition that $f$ is $k$-regular with the condition that $f$ is continuous, then the resulting proposition need not be true, as the following example shows. Define $f$ on the interval $X=[-\sqrt{2},+\infty)$ as follows :

$f(x)=2 x+\sqrt{2}$ if $-\sqrt{2} \leqq x \leqq \sqrt{2}, f(x)=x / 2+5 / \sqrt{2}$ if $x>\sqrt{2}$. $f$ is not $k$-regular, $0<k<1$, and $Y_{f}$ is a singleton where $Y=Q \cap X$. But $f$ has two fixed points.

In the end of this paper, we give another application of Theorem 4, which treat a subject of Kannan's fixed point theorem in metric space.

Theorem 6. Let $X$ be a complete metric space. Let $f$ be a continuous mapping of $X$ into itself such that

$$
d(f(x), f(y)) \leqq \alpha d(x, f(x))+\beta d(y, f(y))+\gamma d(x, y) \quad \text { where } x, y \in X
$$

and $0<\alpha+\beta+\gamma<1,0 \leqq \alpha, 0 \leqq \beta<1,0 \leqq \gamma<1$. Then $f$ has a unique fixed point.

Proof. Let $x, y$ be any point of $X$. In order to complete the proof, we see $x \sim y$. For all $n$ we have

Hence we have

$$
\begin{aligned}
& d\left(f^{n}(x), f^{n+1}(x)\right) \leqq\left(\frac{\alpha+\gamma}{1-\beta}\right)^{n} d(x, f(x)) . \\
& d\left(f^{n}(x), f^{n}(y)\right) \leqq \alpha d\left(f^{n-1}(x), f^{n}(x)\right)+\beta d\left(f^{n-1}(y), f^{n}(y)\right) \\
& +\gamma d\left(f^{n-1}(x), f^{n-1}(y)\right) \text {. }
\end{aligned}
$$

$$
\begin{aligned}
d\left(f^{n+1}(x), f^{n+1}(y)\right) \leqq & \alpha\left(\frac{\alpha+\gamma}{1-\beta}\right)^{n} d(x, f(x))+\beta\left(\frac{\alpha+\gamma}{1-\beta}\right)^{n} d(y, f(y)) \\
& +\gamma d\left(f^{n}(x), f^{n}(y)\right) .
\end{aligned}
$$

By the induction,

$$
\begin{aligned}
d\left(f^{n+1}(x), f^{n+1}(y)\right) \leqq & \left(\sum_{i=0}^{n} \gamma^{i}\left(\frac{\alpha+\gamma}{1-\beta}\right)^{n-i}\right)\{\alpha d(x, f(x))+\beta d(y, f(y))\} \\
& +\gamma^{n+1} d(x, y) .
\end{aligned}
$$

Let $B_{n}=\sum_{i=0}^{n} \gamma^{i}\left(\frac{\alpha+\gamma}{1-\beta}\right)^{n-i}$. We have $\lim _{n \rightarrow \infty} B_{n}=0$. Therefore

$$
d\left(f^{n}(x), f^{n}(y)\right) \rightarrow 0 \quad \text { as } n \rightarrow+\infty .
$$

This implies that $X_{f}$ is a singleton. By Theorem 4 the proof is complete.

Q.E.D.

Remark 3. By Theorem 6, we have the Banach's fixed point Theorem and Kannan's result [7].

Added in proof. Some changes need in Theorem 3 and Theorem 4. $\bar{h}$ has a unique fixed point $\bar{x}$ with a Cauchy sequence $\left\{h^{n}(x)\right\}$, if and only if, $h$ has a unique fixed point. However, we assume that there exists a Cauchy sequence $\left\{h^{n_{i}}(x)\right\}$ for some $x$. In this case, if $X_{h}$ is a singleton, then $h$ has a unique fixed point. Therefore, in this case 
Theorem 1, Lemma 2 and Theorem 5 are valid. Thus Remark 1 and two lines (p. 925, lines 24,25 ) in this paper, should be deleted.

\section{References}

[1] R. D. Anderson: Hilbert space is homeomorphic to the countable infinite product of lines. Bull. Amer. Math. Soc., 72, 515-519 (1966).

[2] T. Homma and S. Kinoshita: On a topological characterization of the dilation in $E^{3}$. Osaka Math. J, 6, 135-144 (1954).

[3] L. S. Husch: A topological characterization of the dilation in $E^{n}$. Proc. Amer. Math. Soc. (to appear).

[4] - : Topological characterization of the dilation and the translation in Frechet spaces. Math. Ann., 190, 1-5 (1970).

[5] S. Kashiwagi and H. Maki: Generalizations of fixed point theorems. Coll. Pap. Math. Soc. Wakayama Univ., 1, 1-9 (1970).

[6] B. Kérekjártó: Topologisch characterisierungen der linearen Abbildungen, Acta Litt. ac. Sci. Szeged, 6, 235-262 (1934).

[7] R. Kannan: Some results on fixed points. Bull. Calcutta Math. Soc., 60, 71-76 (1968). 